\title{
Le commerce de la châtaigne : un nouvel aspect dans l'étude de la différenciation génétique de populations de châtaigniers (Castanea sativa Mill) en France
}

\author{
N Frascaria, M Lefranc \\ Université Paris-XI, URA 121, Bât 362, Laboratoire de systématique et d'écologie végétales, \\ 91405 Orsay Cedex, France
}

(Reçu le 7 novembre 1990; accepté le 20 septembre 1991)

\begin{abstract}
Résumé - Cinq populations de châtaigniers localisées en France ont été analysées à l'aide de 3 marqueurs enzymatiques polymorphes. La distance genetique de Mahalanobis (Anderson, 1958) a été calculée pour chaque paire de populations. À un certain seuil de similarité, 2 groupes de populations apparaissent situés l'un à l'Est, l'autre à l'Ouest de la France. Par ailleurs, une grande similarité génétique semble exister entre des populations distantes de $800 \mathrm{~km}$. Quelques témoignages historiques apportent des éléments de réponse. Le commerce de la châtaigne au cours des siècles semble justifier nos résultats génétiques. Ainsi, il apparaît clairement que l'impact de l'homme a été essentiel dans l'établissement des populations de châtaigniers en France.
\end{abstract}

Castanea sativa Mill / isozyme / distance génétique / commerce de la châtaigne / différenciation génétique

Summary - Chestnut trade: a new aspect in the study of the genetic differentiation of chestnut-tree (Castanea sativa Mili) populations in France. Five chestnut-tree populations located in France have been analysed for 3 polymorphic enzymatic markers. The genetic distance between pairs of populations is evaluated by the technique of Mahalanobis (Anderson, 1958). At a given genetic similarity threshold, 2 geographic groups of populations are observed, one situated in the west, the other in the east of France. Furthermore, a large genetic similarity appears between populations at a distance of more than $800 \mathrm{~km}$. Some historical events give answers. Chestnut trade over the centuries could explain our genetic results. Thus, it appears clearly that human activities have been essential in the establishment of chestnut-tree populations in France.

Castanea sativa Mill / isozyme / genetic distance / chestnut trade / genetic differentiation 


\section{INTRODUCTION}

Espèces majoritairement allogames, les arbres forestiers entretiennent une variabilité qu'il convient d'explorer. L'étude de la répartition de la variabilité génétique d'une espèce au niveau de son aire de distribution est intéressante car l'espèce subit inévitablement des pressions de sélection différentes en fonction des caractéristiques climatiques et édaphiques qui règnent dans chaque type d'environnement. La différenciation génétique entre plusieurs populations, à la suite de phénomènes de migration ou d'isolation, traduit la microévolution que l'on peut définir comme des changements de caractères à l'intérieur d'une espèce (Cuguen, 1986). L'utilisation de marqueurs biochimiques pour l'étude de la variabilité génétique du châtaignier (Castanea sativa Mill) est récente et le nombre de travaux publiés réduit (Malvolti et Fineschi, 1987; Pigliucci et al, 1990). La présente note décrit une partie de la variabilité alloenzymatique de 5 châtaigneraies françaises. Ii s'agit d'une première approche à l'aide d'un petit nombre de mar- queurs protéiques (PGI, IDH, SDH). Nous présenterons le calcul d'une distance génétique particulière obtenue à l'aide de fréquences alléliques : la distance de Mahalanobis (Anderson, 1958).

\section{MATÉRIEL ET MÉTHODES}

\section{Répartition des populations, échantillonnage}

Les 5 populations analysées se répartissent du Limousin aux Cévennes avec une population située en lle-de-France. Les caractéristiques stationnelles et forestières de chacune d'entre elles sont reportées dans le tableau I. II s'agit de taillis de châtaigniers qui ont été cartographiés chez des propriétaires de placettes boisées par I'Institut pour le développement forestier (IDF). La date d'ensouchement de ces taillis est inconnue mais correspond certainement à des plantations du siècle dernier (d'après plusieurs cahiers des charges). Le prélèvement du matériel constitué de rameaux feuillés et destiné à l'analyse enzymatique a été effectué au hasard sur des nombres variables d'individus allant de 36 pour les plus faibles à 117 pour les plus élevés.

Table I. Situation et caractéristiques stationnelles des 5 populations étudiées.

\begin{tabular}{lcccccccccccc}
\hline \multicolumn{2}{l}{ Population Dept } & A & L & I & S & $x$ & Age & n & p & E & e & Sol \\
\hline Dourdan & 91 & A & $2^{\circ} 30^{\prime}$ & $48^{\circ} 35^{\prime}$ & 0,07 & 13 & 16 & 117 & 10 & S & 140 & LP \\
Naillat & 23 & B & $1^{\circ} 29^{\prime}$ & $46^{\circ} 14^{\prime}$ & 0,30 & 19 & 40 & 63 & 10 & SO & 400 & BL \\
St Mariens & 33 & D & $0^{\circ} 24^{\prime}$ & $45^{\circ} 08^{\prime}$ & 1,5 & 25 & 45 & 38 & 0 & 0 & 0 & BL \\
Alzon & 30 & E & $3^{\circ} 27^{\prime}$ & $43^{\circ} 58^{\prime}$ & - & 21 & 32 & 36 & 40 & SE & 750 & BL \\
St Pons & 34 & F & $2^{\circ} 45^{\prime}$ & $43^{\circ} 29^{\prime}$ & - & 17 & 40 & 42 & 10 & SE & 400 & BL \\
\hline
\end{tabular}

dept : département; $A$ : abréviation; $L$ : longitude Est par rapport à Greenwich; $I:$ latitude; $s$ : surface en ha; $x$ : hauteur en $m$ des arbres les plus hauts; $n$ : nombre d'individus considerés; $p$ : pente en \%; $E$ : exposition; $e$ : altitude en $\mathrm{m} ; \quad L P$ : sol lessivé podzolique; $B L$ : sol brun lessivé. 


\section{Techniques d'analyse biochimique et marqueurs utilisés}

La technique d'extraction et le matériel végétal utilisé (bourgeons, très jeunes feuilles) sont ceux préconisés par Malvolti et Fineschi, 1987. Trois systèmes enzymatiques ont été choisis sur la base de critères techniques (bonnes révélations) et génétiques (polymorphes). II s'agit des phosphoglucose isomérases (PGI, EC 5.3.1.9.), des isocitrate déshydrogénases (IDH, EC 1.1.1.42.) et des shikimate déshydrogénases (SDH, EC 1.1.1.25.). Ces enzymes sont mises à migrer sur gel d'amidon par électrophorèse horizontale. Le tampon de migration est décrit par Meinzel et Markert (1968). Les gels après migration sont découpés et révélés selon la technique de Harris et Hopkinson (1976) et de Tanksley et Rick (1980). L'interprétation génétique des zymogrammes a été mise au point par Bonnefoi (1984) ainsi que par Villani et al (1986) et concerne un locus à 2 allèles par enzyme.

\section{Méthodes statistiques}

\section{Distances génétiques}

Nous avons envisagé de calculer pour nos 5 groupes de populations la distance de Mahalanobis. Le calcul de cette distance revient à soumettre les données brutes au filtre de la variation intrapopulation en les exprimant dans la base des vecteurs propres (axes principaux) de la matrice variance-covariance (Hébert et Vincourt, 1985). Cette distance s'écrit :

$$
D^{2}(1, \mathrm{~J})=\left(x_{1}-x_{\mathrm{J}}\right)^{\prime} W^{-1}\left(x_{1}-x_{\mathrm{J}}\right)
$$

où $W$ est la matrice de variance covariance intrapopulation et $x_{1}$ et $x_{J}$ sont les vecteurs observés des populations $l$ et $J$. Bien que rarement utilisée à cette fin, la distance de Mahalanobis peut être envisagée pour le calcul des distances entre fréquences alléliques en considérant ces dernières comme des caractères (Jacquart, 1974).

\section{RÉSULTATS}

\section{Distances génétique (tableau II)}

Trois groupes de populations sont remarqués. Les populations $(A, F)$ et $(B, D)$ sont proches génétiquement entre elles $(0,0316$ et 0,0632$)$. Les 2 groupes de populations restent éloignés entre eux $(0,2212$ et 0,3160 ). La population $E$ reste un peu marginalisée. Bien qu'individualisée du groupe (B,D) $(0,3950$ et 0,4582$)$, elle reste plus proche des populations $A$ et $F$ $(0,1738$ et 0,1422$)$ que ne le sont les populations $B$ et $D(0,2212$ et 0,2844$)$.

\section{DISCUSSION ET CONCLUSION}

À un certain seuil de similarité génétique, 2 groupes de populations semblent apparaître, l'un à l'ouest l'autre à l'est. Ceci témoigne, bien que le nombre de loci et de

Tableau II. Distances génétiques de Mahalanobis pour chaque couple de populations (logiciel de calcul : Stat ITCF).

\begin{tabular}{|c|c|c|c|c|c|}
\hline & $A$ & $B$ & $c$ & $D$ & $E$ \\
\hline A & 0 & & & & \\
\hline B & 0,2112 & 0 & & & \\
\hline$D$ & 0,3160 & 0,0632 & 0 & & \\
\hline$E$ & 0,1738 & 0,3950 & 0,4882 & 0 & \\
\hline$F$ & 0,0316 & 0,2212 & 0,2844 & 0,1422 & 0 \\
\hline
\end{tabular}


populations soit faible, de l'origine génétique certainement différente des 2 lots de populations. Comment expliquer tous ces résultats ? Certains faits historiques vont nous aider à trouver des éléments de réponse. Si la châtaigne correspond à une production strictement localisée, elle n'en constitue pas moins une denrée qui circule, connue et consommée bien au-delà des zones de culture. Entre le $x^{\theta} l^{\ominus}$ et le $X \mid X$ siècles, 2 grands axes de circulation du fruit sont observés (fig 1, Pitte, 1986). Ces 2 courants vont du sud vers le nord. L'un vient d'Italie (et peut-être de plus loin), passe les Alpes et transite à Lyon où il se grossit de la production du Midi de la France. De là, il s'achemine vers Paris et ensuite vers le nord et l'est. L'autre arrive du Portugal et d'Espagne et retrouve à Bordeaux essentiellement la production des pays du Sud-Ouest, de l'Ouest et du Limousin. Par mer, il gagne les côtes d'Angleterre et des Pays-Bas (fig 1) (Champbier, 1560; Estienne et Liebault, 1564; de Serre, 1600; Montesquieu, 1728; Savary des Bruslons, 1741; du Monceau, 1755 in Bruneton-Governatori, 1984). Ces témoignages historiques apportent un élément de réponse intéressant. Le commerce de la châtaigne pourrait justifier complètement les 2 subdivisions de nos populations l'une vers l'est, l'autre vers l'ouest, mettant ainsi en évidence les origines génétiques diverses.

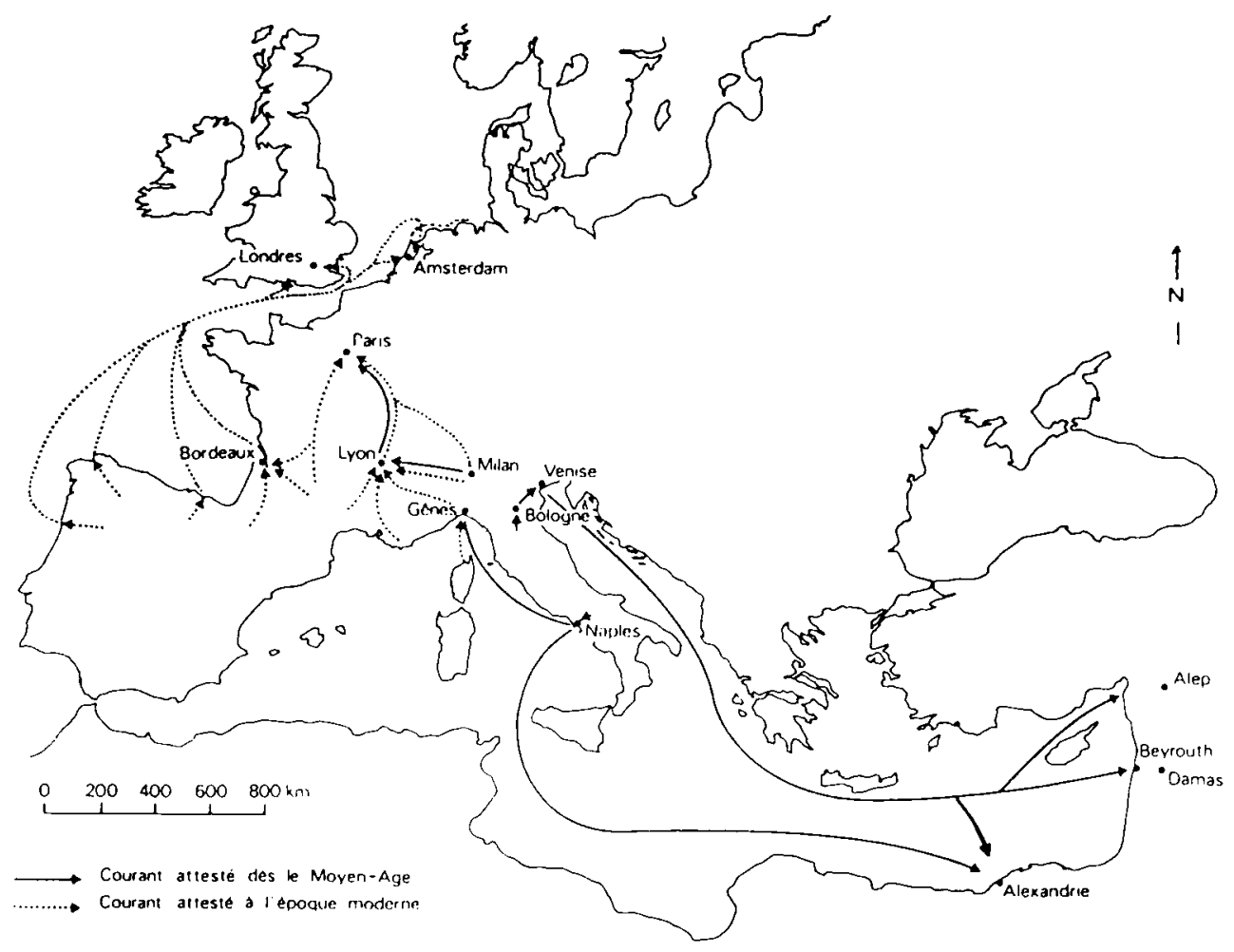

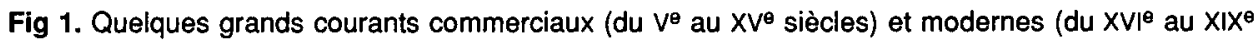
siècles) de châtaignes et marrons (Pitte, 1986). 


\section{REMERCIEMENTS}

Nous remercions $C$ Bourgeois (IDF) pour sa collaboration, JR Pitte (géographe à l'université de Paris-Sorbonne) qui nous a autorisés à utiliser une figure de sa thèse d'état, ainsi que $P H$ Gouyon (professeur à l'université de Paris XI) pour ses remarques lors de la rédaction de cette note.

\section{RÉFÉRENCES}

Anderson TW (1958) An introduction to multivariate statistical analysis Wily $\mathrm{J}$ and Sons. INC, NY, $374 \mathrm{p}$

Bonnefoi C (1984) Étude du polymorphisme enzymatique des populations forestières de châtaignier (Castanea sativa Mill). Thèse en sciences agronomiques, USTL Montpellier, $142 p$

Bruneton-Governatori A (1984) Le pain de bois, éthohistoire de la châtaigne et du châtaignier. Eché Toulouse, $533 p$

Cuguen J (1986) Différenciation génétique interet intrapopulations d'un arbre forestier anémophile : le cas du hêtre (Fagus silvatica $\mathrm{L}$ ).
Thèse docteur en sciences, USTL Montpellier, $83 p$

Hébert $Y$, Vincourt $P$ (1985) Mesures de divergence génétique. 2. Distances calculées sur des critères biométriques. In : Les distances génétiques, estimations et applications, INRA Versailles, 23-39

Jacquart A (1974) Généalogies et distance entre populations. In: Genetic distance (Crow JF Denniston Cn eds) Plenum press, NY, 2340

Malvolti ME, Fineschi S (1987) Analysis of enzyme systems in chestnut (Castanea sativa Mili). Genet Agr 41, 243-256

Meinzel S, Market CL (1967) Malate dehydrogenase isozymes of the marine snail, llyanassa obsoleta. Arch Biochem Biophys 122, 753-765

Pigliucci M, Benetelli S, Villani F (1990) Spatial patterns of genetic variability in Italian chestnut (Castanea sativa). Can J Bot 68, 9, 19621967

Pitte JR (1986) Terres de castanide, hommes et paysages du châtaignier de l'antiquité à nos jours, Fayard, Évreux, $479 p$

Tanksley SD, Rick CM (1980) Isozymic gene linkage map of the tomato, applications in genetics and breeding. Theoret Appl Genet 57, $161-170$ 